Research Article

\title{
Study on the Variation Rule and Characteristics of Pore Water Pressure in the Failure Process of Saturated Rock
}

\author{
Yuezheng Zhang $\mathbb{D}^{1,2}$ \\ ${ }^{1}$ University of Science \& Technology Beijing, Beijing 100083, China \\ ${ }^{2}$ Beijing Key Laboratory of Urban Underground Space Engineering, Beijing 100083, China \\ Correspondence should be addressed to Yuezheng Zhang; yuezheng20053660@163.com
}

Received 4 August 2021; Accepted 1 November 2021; Published 13 November 2021

Academic Editor: Yun Lin

Copyright (c) 2021 Yuezheng Zhang. This is an open access article distributed under the Creative Commons Attribution License, which permits unrestricted use, distribution, and reproduction in any medium, provided the original work is properly cited.

With the development of tunnels and other engineering constructions into the deep strata, rock masses are more prone to dynamic damage such as rock bursts under in situ conditions and excavation disturbances. The pore water in the rock mass will produce pressure changes during this process. According to the relationship between the change of pore water pressure and the development of rock mass damage, the variation rule and precursor characteristics of pore water pressure in the process of rock mass failure can be found. In this paper, through mechanical analysis, the evolution law of pore water pressure during the failure process of saturated rock is obtained. The study found that, in the process of rock failure, the pore water pressure presents three stages of linear growth, transition, and decrease. The rise and fall of pore water pressure are closely related to rock damage and influence each other. Through the observation of pore water pressure during coal mining, it is found that the coseismic effect of pore water pressure is significant. It is proved that there is a close correlation between the evolution of the stress field in the surrounding area of the stope and the change of pore water pressure in the surrounding area under the effect of mining disturbance. During the engineering practice, dynamic monitoring can be carried out on the change of pore water pressure inside the rock mass according to the law, and the precursor information of rock mass instability and failure can be explored.

\section{Introduction}

The presence of pore water in the rock has a significant impact on the physical and mechanical properties of the rock, which is mainly reflected in the strength and failure mode of the rock. And the degree of the influence increases with the increase of rock porosity $[1,2]$. The macroscopic fracture, instability, and failure of the rock after loaded are closely related to the distribution of internal microcracks and the generation, expansion, and penetration of microcracks during deformation. The deformation of the rock has caused the change of its internal pore volume, so the pore water pressure is closely related to the damage of the rock [3-5]. Studies have shown that, during the process of rock failure under pressure, the pore water pressure presents a "rise-decrease" change corresponding to the failure process. According to this finding, the seismic research scholar Che Yongtai and others believe that the water level changes in the aquifer are the anomalous information of earthquake precursors. At the same time, according to the principle of effective stress, the deformation and damage of rock and soil are mainly dominated by effective stress. Under certain conditions of total stress, the pore water pressure and the effective stress will change from one to the other. While the damage development affects the pore water pressure, the pore water pressure also affects the effective stress and then the damage development [6]. Therefore, the interaction relationship between rock damage propagation and pore water pressure changes can be deeply studied to explore the precursor information of critical fracture pore water pressure of rock mass [7-10].

Based on the effective stress, Hooke's law, and damage mechanics principles, this paper theoretically analyzes and establishes the relationship between pore water pressure and rock damage propagation based on the existing experimental research results. The characteristic mode of pore water pressure during the process of elastic deformation, plastic damage, and failure of the rock is obtained. 


\section{Principle of Pore Water Pressure Change Caused by Rock Mass Deformation}

Research shows that the complete process of a seismic event is carried out within a certain range of time and space. The source of the earthquake includes the ruptured body that generates rupture and instability and consumes energy when the earthquake occurs and the surrounding participants that provide energy for the earthquake. In the "relevant area" stratum, the scale of the source is usually much larger than the scale of stratum rupture during the earthquake. The stratum rock mass in a certain area will form a "coordinated seismogenic system" under the action of ground stress. And a certain "structural effect" in the stratum will connect strata on a larger scale to form a "correlation region." In the process of earthquake incubation, a series of characteristics such as ground motion, seismic activity, stress field distribution, and pore water pressure distribution in the relevant area will show a strong "correlation" and "synergy" in a certain spatial range. It also shows different "correlation characteristics" between the physical and mechanical characteristics, spatial scale, stress drop level, source scale, energy consumed and released, and the characteristics of seismic activity related to this seismic activity [11].

When the tectonic stress changes in the ground involve the water-bearing rock and soil layer, the force deformation of the rock and soil will happen. The deformation of the rock and soil will cause the change of the pore volume and then cause the change of the pore water pressure in the rock and soil [12-15]. Zhang and others [16, 17] studied the change pattern of pore water pressure in an ideal confined aquifer under the influence of vertical stress $\sigma_{V}$ changes and obtained the relationship between pore water pressure and stress change:

$$
\Delta H=\frac{n \beta+(1-n) \alpha}{2 \beta \rho g} * \Delta \sigma_{V},
$$

where $\Delta H$ is the change of pore water pressure head caused by the change of the vertical stress of the aquifer $\sigma_{V}, n$ is the porosity of the aquifer, $\alpha$ is the vertical compression coefficient of the aquifer framework, $\beta$ is the volume compression coefficient of water, $\rho$ is the density of water, and $g$ is the acceleration due to gravity.

Che et al. researched that the change of rock volume is mainly caused by the change of porosity, and the change of porosity is related to the nature of the force. Under the action of compressive stress, the porosity becomes smaller; under the action of tensile stress, the porosity becomes larger; under the action of shear stress, the porosity does not change. And the law of sandstone porosity changing with axial compressive stress is

$$
\frac{n_{\sigma}}{n_{0}}=(1-b) 10^{-a \sigma}+b,
$$

where $n_{\sigma}$ is the changed porosity after being stressed, $n_{0}$ is the original porosity, $\sigma$ is the axial stress, $b$ is an index, and $E$ is the ratio after the area is stabilized.
For saturated rock mass, not only the stress of the rock mass but also the pore water pressure will increase during the compression process. If it is a permeable stratum, the excess pore water pressure will dissipate over time and cause the change of groundwater level. The change of well water level during the earthquake is also a macroscopic phenomenon that reflects this law [18].

It can be seen that the stress and deformation of the rock mass will cause damage to the pore water pressure at the same time. The stress and deformation of the rock mass are closely related to the pore water pressure. The change of pore water pressure can be used as earthquake precursor information. To find this precursory information feature, it is necessary to understand the correlation between the damage propagation and the change of pore water pressure during the rock stress process.

\section{Correlation Analysis of Pore Water Pressure and Rock Damage}

3.1. The Model of Pore Water Pressure Change during Rock Failure. There is pore water pressure in the rock with saturated water, and the deformation of the rock and soil is determined by the effective stress. The relationship between effective stress, pore water pressure, and total stress is

$$
\sigma=\sigma^{\prime}+p
$$

In the formula, $\sigma, \sigma^{\prime}$, and $p$, respectively, represent total stress, effective stress, and pore water pressure.

When the rock is compressed under water saturation, the pore water pressure will have a significant impact on the strength and failure mode of the rock. However, there will not be a significant impact on the elastic modulus. The deformation of the water-bearing rock mass causes the change of its pore water volume and then causes the change of pressure in pore water in the rock mass.

Che et al. obtained the pore water pressurepchange curve through triaxial test research on fine sandstone. On this basis, this paper drew the "pore water pressure stress" in the rock closed unit body (without hydraulic contact with the outside world). Comparison chart of "strain" change relationship (Figure 1) shows that the "stressstrain" curve corresponds to the elastic stage, the plastic stage, and the failure stage. The pore water pressure presents $a-b$ linear increase, $b-c$ increase-decrease transition, and c-d decrease.

3.1.1. Pore Water Pressure: $a-b$ Linear Growth Stage. The "stress-strain" curve changes linearly. When the deformation is in the elastic stage, the elastic modulus $E$ remains unchanged, there is no damage inside the rock, and the porosity remains unchanged. Therefore, the increase in pore water pressure is due to the reduction of pore space caused by the elastic deformation of the rock under pressure.

Considering that water is compressible, the compressibility is $\beta$, and the change in pore water pressure is $\Delta p_{1}$, then the change in water volume $\Delta V_{W-1}$ can be expressed as 


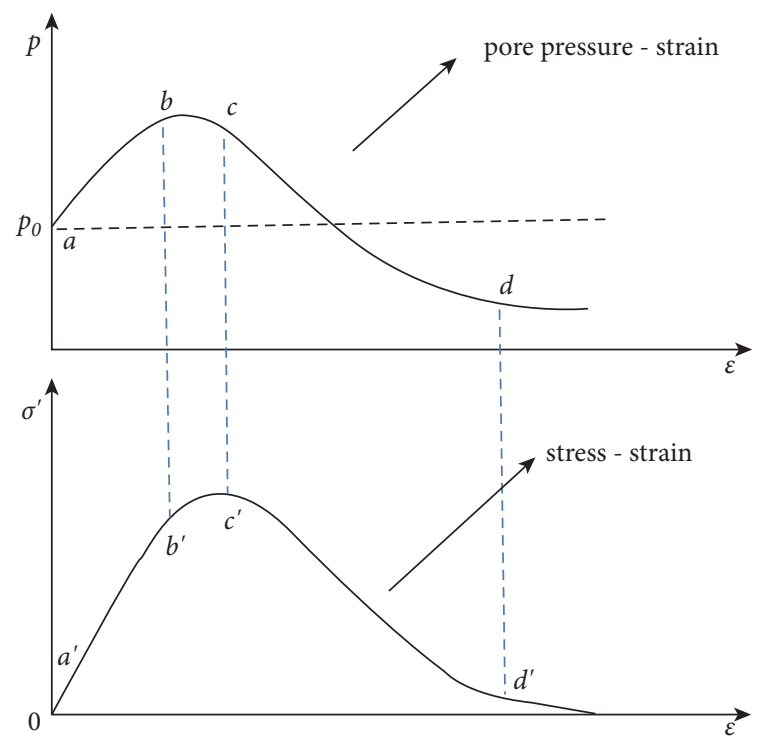

FiguRE 1: "Pore pressure-stress-strain" relation diagram.

$$
\Delta V_{W-1}=-\Delta p \beta
$$

The next step is to find the volume change of the rock $\Delta V$. The rock material is a continuous medium material. According to Hooke's law, the volumetric strain $\theta$ and volumetric stress $\Theta$ in the elastic phase of deformation have the relationship as follows:

$$
\begin{aligned}
\theta & =\frac{1-2 \nu}{E} \Theta, \\
\Delta \theta & =-\frac{(1-2 \nu)}{E} \Delta \Theta \\
& =-\frac{(1-2 \nu)}{E} \Delta\left(\sigma_{x}^{\prime}+\sigma_{y}^{\prime}+\sigma_{z}^{\prime}\right) .
\end{aligned}
$$

Triaxial test under constant confining pressure $\sigma_{y}^{\prime}=\sigma_{z}^{\prime}=$ constant; therefore, $\sigma_{y}^{\prime}=\sigma_{z}^{\prime}=0$; in order to facilitate the analysis, $\Delta \sigma^{\prime}=\Delta \sigma_{x}^{\prime}$; formula (5) can also be expressed as follows:

$$
\Delta \theta=-\frac{(1-2 \nu)}{E} \Delta \sigma^{\prime}
$$

where $\Delta V$ is the volume change of rock, $V$ is the volume of specimen, and the relationship between them is as follows:

$$
\Delta V=\Delta \theta * V
$$

The porosity is $n$ and the relationship between $\Delta V_{W-1}$ and $\Delta V$ is shown in

$$
\Delta V_{W-1}=n \Delta V
$$

Combined with formulas (4), (6), and (7), it can be concluded that

$$
\begin{gathered}
-\beta \Delta p_{1}=-\frac{(1-2 \nu)}{E} n V \Delta \sigma^{\prime}, \\
\Delta p_{1}=\frac{(1-2 \nu)}{E \beta} n V \Delta \sigma^{\prime} .
\end{gathered}
$$

By integrating both sides of formula (10), the relationship between pore water pressure and stress in the elastic compression stage is as follows:

$$
\begin{aligned}
p & =p_{0}+\int_{a^{\prime}}^{\sigma^{\prime \prime}} \frac{(1-2 \nu)}{E \beta} n V d \sigma^{\prime} \\
& =p_{0}+\frac{(1-2 \nu)}{E \beta} n V \sigma^{\prime} .
\end{aligned}
$$

It can be seen from formula (10) that the pore water pressure $p$ is a one-dimensional linear function about the effective stress $\sigma^{\prime}$, so the pore water pressure in the a-b stage is similar to the stress change, showing a linear growth relationship.

3.1.2. Pore Water Pressure: b-c "Increase-Decrease" Transitional Stage. The "stress-strain" curve shows that the rock deformation enters the plastic stage, and the pore water pressure increases slowly and then decreases after reaching the peak. Similarly, the change of pore water pressure is caused by the change of pore space. At this stage, the change of pores in the rock is mainly composed of two parts. (1) The elastic compression of the solid framework causes the reduction of pores. This part of the change is consistent with the a-b elastic stage. (2) Damage occurs inside the rock, and the deformation changes from elastic compression to expansion. At the same time, the microcracks continue to develop, the damage increases, the porosity of the rock $n$ increases, and the effective elastic modulus $\widetilde{E}$ decreases. The sum caused by elastic deformation can be calculated according to formulas (8) and (11). Here, we will focus on the change $\Delta p_{2}$ in pore water pressure caused by plastic deformation.

Rock material is a continuous mechanical medium. Under the action of external load, it will undergo several stages of compaction, elasticity, plasticity, and failure. Reflecting on the damage is that the internal damage produces and develops into a macroscopic crack, which eventually leads to the rupture of the internal structure. It is assumed here that after the rock material is stressed to a certain level, uniform damage occurs, and the change of damage is related to the effective stress acting on the rock structure. Rabonov first introduced the variable corresponding to the continuous variable, namely, the damage variable $D$ [19-21],

$$
D=\frac{A^{*}}{A},
$$

where $A$ is the force-receiving area when there is no initial damage and $A^{*}$ is the damaged area after damage.

First of all, pay attention to the pore change caused by damage. The volume change of pore water $\Delta V_{W-2}$ is proportional to the damage $D\left(\sigma^{\prime}\right)$, and the proportional coefficient is $m$ : 


$$
\begin{aligned}
\Delta V_{W-2} & =m * V * D\left(\sigma^{\prime}\right), \\
-\beta \Delta p_{2} & =m V D\left(\sigma^{\prime}\right), \\
\Delta p_{2} & =-\frac{m V}{\beta} D\left(\sigma^{\prime}\right), \\
p & =p_{o}+\frac{(1-2 \nu)}{E \beta} V \sigma^{\prime}+\int_{b^{\prime}}^{c^{\prime}} \frac{m V}{\beta}\left(\frac{\widetilde{E}\left(\sigma^{\prime}\right)}{E}-1\right) \mathrm{d} \sigma^{\prime}, \\
p & =p_{o}+\frac{(1-2 \nu)}{E \beta} V \sigma^{\prime}+\int_{b^{\prime}}^{c^{\prime}} \frac{m V}{\beta}\left(\frac{\widetilde{E}\left(\sigma^{\prime}\right)}{E}-1\right) \mathrm{d} \sigma^{\prime} .
\end{aligned}
$$

Formula (17) is the constitutive relationship between pore water pressure and stress in the plastic deformation stage. Damage variables are generally difficult to see from the stress-strain curve. In order to further express this constitutive relationship intuitively, according to the principle of strain equivalence proposed by Lemaitre, the effective stress $\sigma^{\prime}$ and damage variable $D\left(\sigma^{\prime}\right)$ have the following constitutive relationship:

$$
\sigma^{\prime}=E(1-D) \mathcal{\varepsilon}
$$

In the formula, $\sigma^{\prime}, D, E$, $\varepsilon$, respectively, represent effective stress, damage variable, rock elastic modulus, and strain. Introducing the effective modulus of elasticity $\widetilde{E}\left(\sigma^{\prime}\right)=E\left(1-D\left(\sigma^{\prime}\right)\right)$, the damage variable can also be expressed as

$$
\begin{aligned}
D\left(\sigma^{\prime}\right) & =1-\frac{\widetilde{E}\left(\sigma^{\prime}\right)}{E} \\
p & =p_{o}+\frac{(1-2 \nu)}{E \beta} V \sigma^{\prime}+\int_{b^{\prime}}^{c^{\prime}} \frac{m V}{\beta}\left(\frac{\widetilde{E}\left(\sigma^{\prime}\right)}{E}-1\right) \mathrm{d} \sigma^{\prime} .
\end{aligned}
$$

It can be seen intuitively from formula (20) that $\widetilde{E}\left(\sigma^{\prime}\right)$ reflects the change in the slope of the "stress-strain" curve. As $\widetilde{E}\left(\sigma^{\prime}\right)$ continues to decrease, $\Delta V_{W-2}$ continues to increase, and $\Delta p_{2}$ causes a continuous decrease. At that time, when $\left|\Delta p_{1}\right|>\left|\Delta p_{2}\right|, \Delta p>0$, and $p$ increase; at that time, when $\left|\Delta p_{1}\right|=\left|\Delta p_{2}\right|, \Delta p=0, p$ reaches the maximum value; when $\left|\Delta p_{1}\right|<\left|\Delta p_{2}\right|, \Delta p<0, p$ decrease, so the pore water pressure presents a transition characteristic of "increasedecrease.”

3.1.3. Pore Water Pressure: $c$ - $d$ Decreasing Stage. When the stress reaches the peak, the cracks in the rock will be further penetrated, and the rock will enter the failure stage. The internal pore space increases, and the pore water pressure continues to decrease. The relationship between the pore water pressure $p$ and $\sigma^{\prime}$ the plastic deformation stage after the peak failure deformation stage is the same as that of the plastic deformation stage:

$$
p=p_{0}+\frac{(1-2 \nu)}{E \beta} V \sigma^{\prime}-\int_{b^{\prime}}^{c^{\prime}} \frac{m V}{\beta} D\left(\sigma^{\prime}\right) \mathrm{d} \sigma^{\prime} .
$$

At this stage, due to the sudden change of failure and deformation, the pore water pressure continues to decrease, and the nonlinear change is more obvious. When the rock is completely destroyed, the pore space no longer changes, and the pore water pressure will remain at a certain value, which is less than the initial pore water pressure value, $p \leq p_{0}$.

3.2. Precursor Characteristics of Critical Fracture of Pore Water Pressure. Through the analysis of the change relationship of pore water pressure in the process of rock force change, change relationship of stress, damage, and pore water pressure at each stage can be obtained, as shown in Table 1.

Analysis of the change mechanism of pore water pressure during the stress process shows that pore water pressure is closely related to stress and rock damage. Pore water pressure reflects the characteristics of stress and deformation of rock at various stages of loading. The continuous increase of the pore water pressure in the closed rock mass unit reflects the elastic phase of the increased force, and the rock can be regarded as "safe"; when the pore water pressure continues to increase and the "inflection point" decreases, the rock mass causes plastic damage and the force will reach the limit. The change in pore water pressure is a precursor to the occurrence of the primary failure of the rock.

\section{The Characteristics of Pore Water Pressure Crack Precursor Information and Its Influence on the Development of Rock Damage}

The change of pore water pressure also has an impact on the damage development. According to the principle of effective stress (see formula (3)), during the damage, the pore space inside the rock becomes larger, and the pore water pressure decreases. When the total stress $\sigma$ remains constant, the pore water pressure $p$ decreases, which in turn causes an instantaneous increase in the effective stress and intensifies the development of damage cracks in the rock. The development of damage cracks in turn causes the pore water pressure to continue to decrease. The interaction of the two accelerates the ultimate rock failure. It can be seen that the development of rock damage affects the change of pore water pressure, and the change of pore water pressure also affects the development of rock damage. The two influence each other $[22,23]$.

The underground engineering rock mass is a spatial continuous medium, and the stress transmission is continuous. When the local rock mass is damaged due to the stress and energy concentration caused by excavation disturbance, the formation of rock mass in the surrounding area will also produce corresponding stress changes. And the pore water pressure will increase and decrease alternately. The study of the pore water pressure change can be used to 
TABLE 1: The mode of "stress-injury-pore pressure."

\begin{tabular}{lccc}
\hline & Elastic stage & Plastic stage & Destruction stage \\
\hline Stress & Linear growth & Curve growth & Decline \\
Damage & None & Development & Rapid development \\
Pore water pressure & Linear growth & Growth-decrease transition & Rapid decline \\
\hline
\end{tabular}

analyze its correlation with the rock failure process of adjacent related areas [24, 25].

The test results show that the law of pore water pressure change and permeability can effectively reflect the different stages of the internal damage development of the rock mass [26], the sharp decrease in pore water pressure, and the steep increase in permeability in the CD stage before the main fracture. This is the intuitive information reflection of the changes in the internal physical properties of the rock material. In actual engineering rock masses, when the pore water pressure in the rock mass gradually decreases or sharply decreases from the stable value and the permeability gradually increases or steeply increases from the stable value, large-scale damage and destruction occur inside the rock mass. It will enter a state of instability, which is the precursor information of rock mass rupture. This will provide a new way to predict rock bursts and rock bursts caused by excavation of chambers in rock masses. In actual engineering, it is difficult to measure the permeability inside the rock mass in real time. The pore water pressure monitoring technology developed in the study of seismic monitoring and prediction is becoming more and more mature, which provides an important technical basis for realizing the monitoring of pore water pressure in the rock mass. It is a feasible method to predict rock mass failure by monitoring the change of pore water pressure to predict the deformation of the rock mass in the area.

With the development of technology, the current monitoring accuracy of deep pore water pressure can reach the Pa level. The comprehensive deep borehole observation system popularized in earthquakes can observe information such as formation strain, pore water pressure, ground tilt, and even solid tide, which provides a technical basis for the application in tunnels and mine engineering [27, 28].

\section{The Effect of Pore Water Pressure in the Formation in the Relevant Area Caused by Rock Bursts}

5.1. Engineering Background. In order to verify the relationship between the change of pore water pressure and the failure of the formation rock mass, the study selected the Yanbei Coal Mine located in the Huating Coalfield in Gansu Province, China, which is frequently subjected to rock bursts, as the engineering background to observe the change of pore water pressure before and after rock burst occurs during mining [29].

The Huating Coalfield is located at the eastern foot of the Liupan Mountains, the southwestern edge of the Ordos block, or the southern end of the fault fold belt on the western edge. This area happens to be located at the intersection of the large fault on the western edge of Liupan Mountain, the thrust belt on the western edge of the Ordos Basin, and the Qingtongxia-Guyuan fault, with a complex geological environment. The Huating Coalfield presents a spinning compound asymmetric syncline structure with a "south-east structure, gentle east and steep west, wide middle, and convergent north and south sections," as shown in Figure 2.

The Huating Coalfield is mainly controlled by horizontal tectonic stress, with folds as the main structure and faults as the auxiliary structure. The thickness and dip of the coal seam change along the direction and tendency of the folds, and the fold structure plays a major role in controlling the transformation, destruction, and shaping of the existing coal measures.

Coal seam mining changes the stress distribution characteristics, causing local rock mass stress concentration, and the surrounding rock accumulates a large amount of elastic energy, which is the main factor that induces the appearance of strong rock pressure in the Yanbei and Huating mining areas. The coal seam is a very thick coal seam with a thickness between $30 \mathrm{~m}$ and $40 \mathrm{~m}$. The upper layer is first mined, and then the lower layer is mined. The mining depth reaches $800 \mathrm{~m}-1000 \mathrm{~m}$. The continuous deep mining of Huating Coal Mine and Yanbei Coal Mine in Huating Coalfield for many years has changed the original structural stress distribution characteristics, causing local stress concentration and increased energy storage [30,31]. The direction of the working face of the mining area is close to the north-south direction, which is consistent with the direction of the fold structure axis, and is perpendicular to the direction of the maximum principal stress near the eastwest in the area.

Yanbei Coal Mine is affected by deep mining and regional structure. Mine earthquakes often occur in the syncline axis, especially in structural change areas, near faults, coal seam dip changes, coal seam buckling, and tectonic stress zones. The 250203 and 250204 working faces passed through the shaft and wings of the fold structure during the mining process, and the rock pressure appeared very obvious.

In order to quantitatively observe the quantitative relationship between mine earthquake disaster source rock mass and regional structure, stress environment, mine depth, mining volume, mining intensity, and other factors in the process of mine earthquakes, the RET-2A high-precision pore water pressure gauge with an accuracy of $10^{-9}-10^{-10}$ can be used to observe the microdynamic change process of pore water pressure in the crust. The observation site should be installed on the "force chain structure" of the seismogenic system. The 


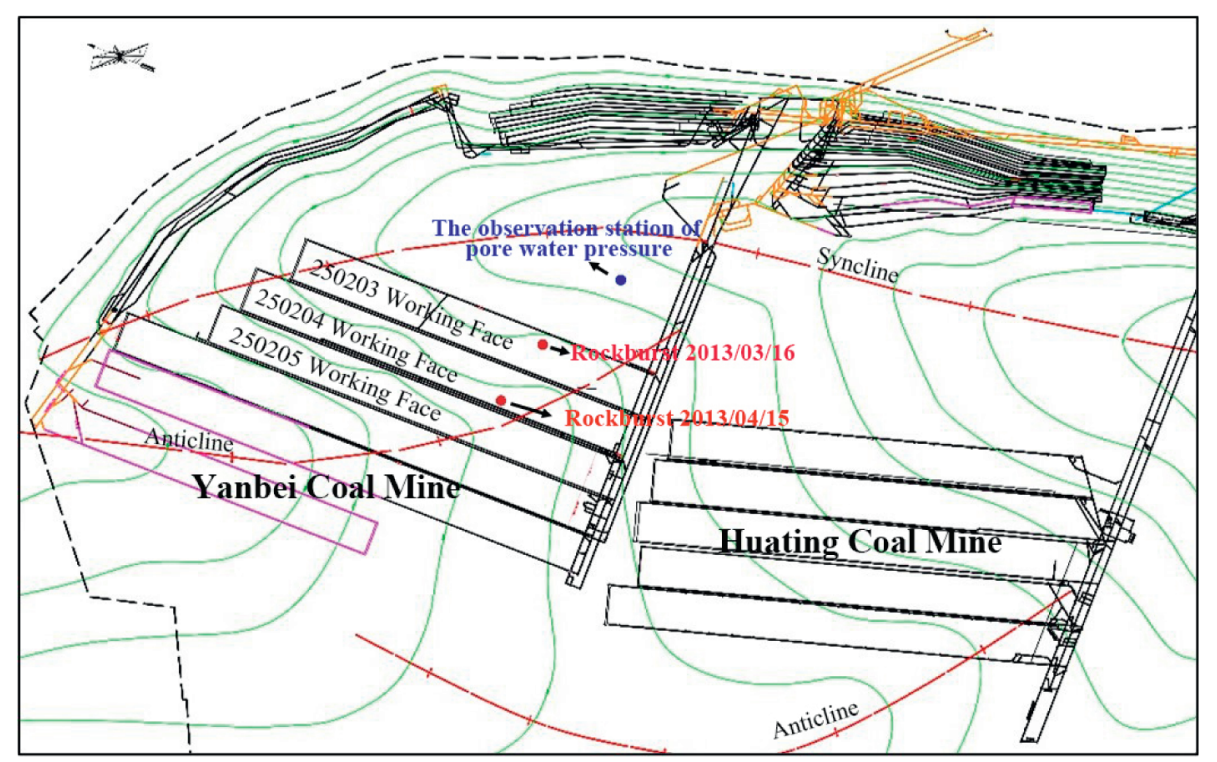

Figure 2: Project overview, location of observation station, and rockburst events.

measurement points and observation characteristic quantities should be jointly arranged according to different characteristic areas such as the core area of the mine earthquake disaster source body, the energy release area, and the surrounding "related areas," as shown in Figure 2. The Yanbei Coal Mine's pore water pressure observation point is installed in the coal seam floor rock at the syncline axis [32, 33].

5.2. Analysis of Measured Data. After the completion of the observatory, continuous observation data of pore water pressure from March 15 to April 19, 2013, were obtained. As shown in Figure 3, the time history data curve of pore water pressure has a significant solid tidal change background, indicating that the expected observation accuracy has been achieved and the equipment is working normally. Due to the high accuracy, in order to obtain an intuitive change rule, no calibration was performed during the analysis process, and the original monitoring data were selected for analysis. During this period of time, mining was carried out at the 250203 working face, and severe rock bursts occurred at 13 : 23 2013/3/16 and 22:28 2013/4/15, which had a serious impact on production. The information of the two rockburst events is shown in Table 2.

As shown in Figure 3, the data obtained by the pore water pressure observation station show that, at the same time that the two rock bursts occurred, the pore water pressure has undergone significant synergistic changes, resulting in a significant coseismic effect. It is mainly manifested as a step increase in pore water pressure, but the magnitude of the change is very small, and the impact of the solid tidal stress is an order of magnitude. This microdynamic change can only be measured by highprecision equipment. From the perspective of the change trend of pore water pressure before and after the occurrence of rockburst, due to the impact of mining, the pore water pressure in the surrounding area has also changed up and down, reflecting that the stress field and pore water pressure in this area are all synergistic variety. This change is mainly manifested as a kind of microdynamic effect, which also shows that the strata in a certain range around the stope and the stope and the rock mass of the seismic source rupture are a coordinated system. The adjustment process of energy accumulation and release in the system can be obtained by observation, and the magnitude is equivalent to that of the Earth tide effect [34].

Since the selection of the observation station is far from the working faces and the location where rock bursts occur, the change of pore water pressure obtained changes with the change of the stress field in the adjacent area of the closed rock mass. This change presents obvious characteristics of microdynamic changes, which proves that there is a close correlation between the evolution of the stress field in the surrounding area of the stope under mining disturbance and the change of the pore water pressure in the surrounding area. Mining dynamic disasters, such as rockburst and mine earthquakes, seriously threaten the safety of the mine. The breeding and inducing processes are the accumulation of factors such as stress and energy, which cause the surrounding rock of the stope to locally reach or exceed the critical state, rupture, and expand to evolve, forming earthquake source or disaster source. The emergence and evolution processes reflect the coaction process of multiple factors such as overburden and stratum structure, original stress field, and mining disturbance and also reflect the synergy mechanism and state evolution process of multiple physical mechanics fields on multiple spatial scales. 


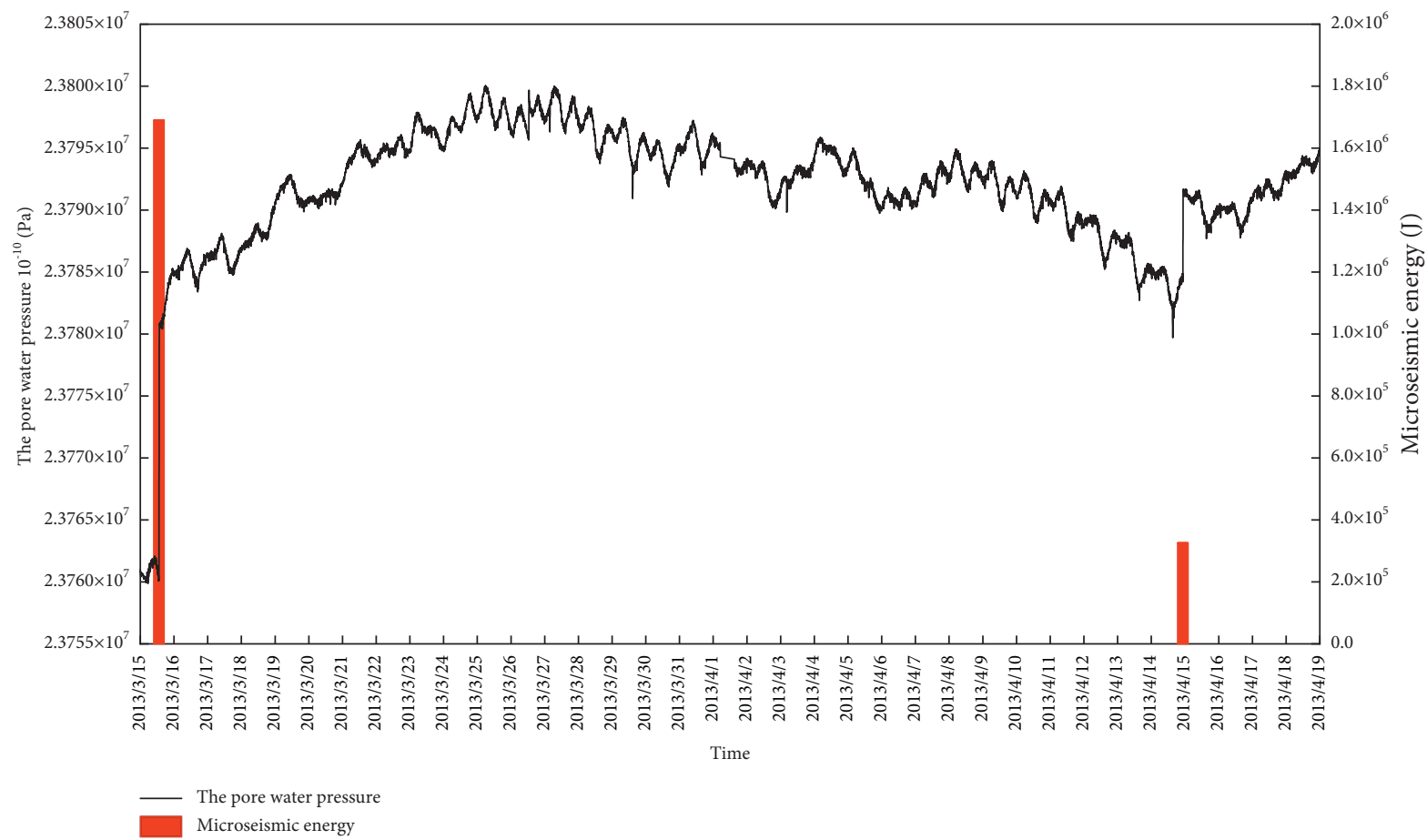

FIgURE 3: Time history curve of pore water pressure.

TABLE 2: Information table of rockburst events.

\begin{tabular}{lccr}
\hline Time & $\begin{array}{c}\text { Distance to observing } \\
\text { station }(\mathrm{m})\end{array}$ & $\begin{array}{c}\text { Microseismic } \\
\text { energy }\end{array}$ & \multicolumn{1}{c}{ Damage } \\
\hline $\begin{array}{l}2013 / 3 / 16 \\
13: 23\end{array}$ & 527.5 & $1.69 E * 10^{6} \mathrm{~J}$ & $\begin{array}{c}\text { The roadway is deformed, the support is damaged, the top sink is } 200-300 \mathrm{~mm}, \\
\text { the bottom drum is 200-500 mm, } 40 \text { anchor cables are broken, and the repair } \\
\text { work volume is about } 812 \mathrm{~m}^{3}\end{array}$ \\
$\begin{array}{l}2013 / 4 / 15 \\
22: 28\end{array}$ & 886.6 & $\begin{array}{r}\text { Deformation of the roadway occurred in the transportation lanes and material } \\
\text { lanes on both sides of the working face, the bottom drum was } 200 \mathrm{~mm}-600 \mathrm{~mm} \text {, } \\
\text { and the repair works amounted to } 353 \mathrm{~m}^{3}\end{array}$ \\
\hline
\end{tabular}

\section{Conclusions and Prospects}

(1) In an ideally closed saturated rock unit, the pore water pressure is closely related to the expansion of internal damage. The pore water pressure presents a linear increase, a growth-decrease transition, and a decrease process and reaches a stable value after the rock is completely destroyed.

(2) Based on the analysis of Hooke's law and the principle of damage mechanics, the analytical model between pore water pressure changes and stress changes at various stages is obtained. The "increasedecrease transition" change of pore water pressure is the precursor information of rock fracture.

(3) According to the analysis of the effective stress principle, the pore water pressure and the damage development interact and are related to each other. The damage expansion causes the pore water pressure to decrease, and the decrease of the pore water pressure promotes the damage development.
(4) At present, the monitoring technology of pore water pressure has been relatively maturely applied in seismic monitoring research. On this basis, monitoring the changes of pore water pressure inside the rock mass in deep mining projects can predict dynamic disaster of instability and destruction of rock bursts and other rock masses.

(5) Through the observation of the pore water pressure in the relevant area during the coal mining process, the response of the pore water pressure in the relevant area before and after the occurrence of rockburst in the mine was successfully obtained. The coseismic effect is obvious, which proves that the gestation and induction process of large-scale structural rock bursts or mine shocks is far greater than the range of traditional stope pressure and mining influence. Analyzing the stress field between the rockburst source and the "relevant area" and its multifield correlation is the correct way to reveal the mechanism of the initiation and induction of mine earthquakes. 


\section{Data Availability}

The data used to support the findings of this study are available from the corresponding author upon request.

\section{Conflicts of Interest}

The authors declare that they have no conflicts of interest.

\section{Acknowledgments}

This work was supported by the National Key Research and Development Program of China under Grant No. 2016YFC0600800, the Major Scientific and Technological Innovation Projects in Shandong Province under Grant No. 2019SDZY02, and Fundamental Research Funds for the Central Universities (FRF-BD-20-01B and FRF-TP-19026A2).

\section{References}

[1] T. H. Yang, C. A. Tang, W. C. Zhu, and Q. Y. Feng, "Coupling analysis of seepage and stresses in rock failure process," Chinese Journal of Geotechnical Engineering, vol. 23, no. 4, pp. 489-493, 2001.

[2] X. Li, K. Peng, J. Peng, and H. Xu, "Effect of cyclic wettingdrying treatment on strength and failure behavior of two quartz-rich sandstones under direct shear," Rock Mechanics and Rock Engineering, vol. 1, no. 8, 2021.

[3] Y. P. Qin, J. F. Zhang, and L. Wang, "Preliminary discussion on theoretical model of rock damage mechanics," Chinese Journal of Rock Mechanics and Engineering, vol. 22, no. 4, pp. 646-650, 2003.

[4] Y. P. Qin, W. B. Sun, and L. Wang, Analysis On damage mechanics model of rock, vol. 22, no. 5, pp. 702-705, 2003.

[5] J. Yu, H. Li, X. Chen, Y. Cai, N. Wu, and K. Mu, "Triaxial experimental study of associated permeability-deformation of sandstone under hydro-mechanical coupling," Chinese Journal of Rock Mechanics and Engineering, vol. 32, no. 6, pp. 1203-1213, 2013.

[6] Y. T. Che, H. N. Yang, and Z. L. Zhang, "Hydrodynamic simulation test and research on the formation process of groundwater microbehavior," Earthquake, vol. 9, no. 5, pp. 40-49, 1989.

[7] Q. Yin, J. Y. Wu, C. Zhu, M. He, Q. Meng, and H. Jing, "Shear mechanical responses of sandstone exposed to high temperature under constant normal stiffness boundary conditions," Geomechanics and Geophysics for Geo-Energy and GeoResources, vol. 7, no. 2, 2021.

[8] K. Sun, L. M. Fan, Y. C. Xia et al., "Impact of coal mining on groundwater of Luohe formation in Binchang mining area," International Journal Coal Science Technol, vol. 8, no. 1, pp. 88-102, 2021.

[9] G. D. Cui, W. X. Wang, B. Dou, and Y. Liu, "Geothermal energy exploitation and power generation via a single vertical well combined with hydraulic fracturing," Journal of Energy Engineering, vol. 148, 2022.

[10] M. Z. Gao, J. Xie, J. Guo, Y. Lu, Z. He, and C. Li, "Fractal evolution and connectivity characteristics of mining-induced crack networks in coal masses at different depths," Geomechanics and Geophysics for Geo-Energy and Geo-Resources, vol. 7, no. 1, 2021.
[11] L. Li, F. M. Li, Y. Zhang, D. Yang, and X. Liu, "Formation mechanism and height calculation of the caved zone and water-conducting fracture zone in solid backfill mining," International Journal Coal Science Technol, vol. 7, no. 1, pp. $208-215,2020$.

[12] Y. S. Li Yong, "The main features of groundwater level changes caused by tectonic stress," Northwestern Seismological Journal, vol. 1, no. 1, pp. 16-22, 1979.

[13] J. Zhu and J. W. Liang, "Effects of dynamic soil-tunnel interaction on seismic soil pressures and pore pressures," Journal of Natural Disasters, vol. 27, no. 6, pp. 66-74, 2018.

[14] C. Zhu, M. C. He, X. H. Zhang, Z. Tao, Q. Yin, and L. Li, "Nonlinear mechanical model of constant resistance and large deformation bolt and influence parameters analysis of constant resistance behavior," Rock and Soil Mechanics, vol. 42, no. 7, pp. 1911-1924, 2021.

[15] Z. G. Yi, Z. H. Qiu, and M. Song, "Analysis of data of digitalized borehole strain observation in capital area," Journal of Geodesy and Geodynamics, vol. 26, no. 3, pp. 53-58, 2006.

[16] Z. D. Zhang, "Comparison and analysis of observational water-level tide of artesian well and gravity solid tide," Earthquake, vol. 11, no. 2, pp. 47-52, 1991.

[17] Z. D. Zhang, J. H. Zheng, C. G. Feng, and G. C. Zhang, "The comparison of the three methods of inversion of stress changes of aquifers using step changes of well level," North China Earthquake Sciences, vol. 11, no. 1, pp. 39-44, 1993.

[18] Z. D. Zhang, Q. G. Liu, and J. Geng, "Stress variation of well aquifer inverted by water level behaviors of pressure well," South China Journal of Seismology, vol. 19, no. 1, pp. 37-42, 1999.

[19] X. Y. Zhang, H. N. Ruan, and C. H. Jia, "Research development of the rock's damage theory," Sichuan Building Science, vol. 36, no. 2, pp. 134-138, 2010.

[20] G. Feng, Y. Kang, X. C. Wang, Y. Hu, and X. Li, "Investigation on the failure characteristics and fracture classification of shale under Brazilian test conditions," Rock Mechanics and Rock Engineering, vol. 53, no. 7, pp. 3325-3340, 2020.

[21] G. D. Cui, L. H. Yang, J. C. Fang, Z. Qiu, Y. Wang, and S. Ren, "Geochemical reactions and their influence on petrophysical properties of ultra-low permeability oil reservoirs during water and CO2 flooding," Journal of Petroleum Science and Engineering, vol. 203, 2021.

[22] Y. W. Liu, L. Q. Xu, and D. X. Yang, "Pore pressure diffusion characteristics of Longtan reservoir-induced-earthquake," Chinese Journal of Geophysics, vol. 54, no. 4, pp. 1028-1037, 2011.

[23] S. L. Liu, Z. N. Li, Y. Yang, J. Shen, Y. Huang, and S. Xu, "Preliminary analysis of the digital observation data quality of borehole body strain in Fujian province," South China Journal of Selsmology, vol. 27, no. 4, pp. 79-87, 2007.

[24] Q. Wang, M. C. He, S. C. Li et al., "Comparative study of model tests on automatically formed roadway and gob-side entry driving in deep coal mines," International Journal of Mining Science and Technology, vol. 31, no. 4, pp. 591-601, 2021.

[25] Q. Wang, Y. Wang, M. C. He et al., "Experimental research and application of automatically formed roadway without advance tunneling," Tunnelling and Underground Space Technology incorporating Trenchless Technology Research, vol. 114, no. 3, 2021.

[26] Y. Z. Zhang and H. G. Ji, "Pore water pressure change-related characteristic and its critical rupture precursor of rock under triaxial compression," Chinese Journal of Engineering, vol. 37, no. 4, pp. 399-406, 2015. 
[27] D. Issei and K. Toshitaka, "Relationship between earthquakeinduced excess pore water pressure and strong ground motion observed in a monitored fill slope," Engineering Geology, vol. $266,2020$.

[28] C. J. Meng, X. W. Wang, S. L. Zhang, and S. Y. Yang, “Analysis of shortleveling anomaly in linfen seismic station based on the groundwater change," Journal of Geodesy and Geodynamics, vol. 40, no. 11, pp. 1206-1210, 2020.

[29] L. Y. Zhao, H. Zhang, and X. F. Liu, "Study on seismicity characteristics and mechanism in pingliang area Gansu province," Northwestern Seismological Journal, vol. 31, no. 2, pp. 186-190, 2009.

[30] H. Jiang, Research on Fracture Characteristic of Surrounding Rock and the Relationship with Microseismic Actibity in Synclinal axis Region, Beijing University of science and technology, Beijing, China, 2020.

[31] F. Chen, A. Y. Cao, L. M. Dou, and C. Wang, "Method of coal burst hazard assessment based on region division and identification of main impact factors," Journal of China Coal Society, vol. 43, no. 3, pp. 607-615, 2018.

[32] Z. H. Qiu and Y. L. Shi, "Relationchip between theoretic magnitude and longest propagation Distance of seismic strain change," Journal of Geodesy and Geodynamics, vol. 24, no. 2, pp. 99-105, 2004.

[33] Y. T. Che, J. Z. Yu, Z. F. Ma, and Q. Wang, "Mining earthquakes and anomalous response of groundwater level in deep well," Earthquake, vol. 17, no. 1, pp. 61-66, 1997.

[34] M. Gao, J. Xie, Y. Gao et al., "Mechanical behavior of coal under different mining rates: a case study from laboratory experiments to field testing," International Journal of Mining Science and Technology, vol. 31, no. 5, pp. 825-841, 2021. 